\title{
PILOT TOXICOKINETIC STUDY AND ABSOLUTE ORAL BIOAVAILABILITY OF THE FUSARIUM MYCOTOXIN ENNIATIN B1 IN PIGS
}

\author{
Mathias Devreese, Patrick De Backer, Siska Croubels
}

Department of Pharmacology, Toxicology and Biochemistry, Faculty of Veterinary Medicine, Ghent University, Salisburylaan 133, B-9820 Merelbeke

Fusarium fungi frequently infest crops in temperate regions such as Western Europe and North America. They can produce a wide range of mycotoxins, including several extensively studied compounds such as trichothecenes, zearalenone and fumonisins. They are also capable of producing other less well-known mycotoxins like enniatins (ENNs) (enniatin (ENN) A, A1, B and B1) and beauvericin (BEA). ENNs are cyclic hexadepsipeptides consisting of alternating $D$ - $\alpha$-hydroxyisovaleric acids and L-methyl-amino acids. Over the last decade, ENNs were found to be common contaminants of grains, maize and other feedstuffs. Several in vitro toxicity studies elucidated their antibacterial, antifungal, antihelmintic, insecticidal and herbicidal potency. Also their cytotoxic effect on a variety of cell types was demonstrated previously. However, the biological activity of ENNs has only been tested in few in vivo studies, demonstrating low acute toxicity, and there are no cases of mycotoxicosis in humans and animals reported.

The aim of present study was to reveal the toxicokinetic properties and absolute oral bioavailability of enniatin B1 in pigs. Five pigs were administered this Fusarium mycotoxin per os and intravenously in a two-way cross-over design. The toxicokinetic profile fitted a two-compartmental model. Enniatin $B 1$ is rapidly absorbed after oral administration $\left(T_{1 / 2 a}=0.15 h, T_{\max }=0.24 h\right)$ and rapidly distributed and eliminated as well $\left(T_{1 / 2 \text { ela }}=0.15 \mathrm{~h} ; \mathrm{T}_{1 / 2 \mathrm{el} \beta}=1.57 \mathrm{~h}\right)$. The absolute oral bioavailability is high $(90.9 \%)$, indicating a clear systemic exposure. After intravenous administration, the mycotoxin is distributed and eliminated rapidly $\left(T_{1 / 2 \mathrm{el} \alpha}=0.15 \mathrm{~h} ; \mathrm{T}_{1 / 2 \mathrm{el} \beta}=1.13 \mathrm{~h}\right)$, in accordance with oral administration.

Future research should focus on elucidating the phase I and II metabolisation pathways of ENNs and the toxicity of these metabolites. Next, toxicokinetic studies of ENN B1, and other ENNs should be performed in other animal species to compare mycotoxin and species dependent differences in toxicity and sensitivity. Finally, the present study may serve as a model for humans, indicating that systemic exposure of ENNs after oral intake of contaminated food cannot be neglected. 